\title{
Exact solution for the non-equilibrium attractor in number-conserving relaxation time approximation
}

\author{
Michael Strickland and Ubaid Tantary \\ Department of Physics, Kent State University, \\ Kent, OH 44242 United States \\ E-mail: mstrick6@kent.edu, utantary@kent.edu
}

\begin{abstract}
We extend previous studies of the conformal $0+1 \mathrm{~d}$ kinetic non-equilibrium attractor in relaxation time approximation by enforcing number conservation through the introduction of a dynamical fugacity (chemical potential). We derive two coupled integral equations for the effective temperature and fugacity which are then solved numerically to obtain the exact solution. The resulting solutions exhibit convergence to a unique nonequilibrium attractor when the scaled moments of the distribution function are plotted as a function of the rescaled time $\bar{w}=\tau / \tau_{\text {eq }}$. This occurs even though the system is out of chemical equilibrium at late times. In addition, compared to the case where number conservation was not imposed, we find that the moments converge to their respective attractors more quickly, particularly for moments with $m=0$. Finally, we compare the resulting attractor moments with predictions from different hydrodynamic frameworks.
\end{abstract}

KeYwORDs: Heavy Ion Phenomenology

ArXiv EPrint: 1903.03145 


\section{Contents}

1 Introduction 1

2 Setup 3

2.1 Boost-invariant variables 4

2.2 Boost-invariant form of the kinetic equation 4

3 Exact solution for the distribution function 5

3.1 Initial distribution 5

3.2 General moments of the distribution function 5

$\begin{array}{ll}3.3 & \text { Integral equation obeyed by a general moment }\end{array}$

4 Results $\quad 7$

$\begin{array}{lll}4.1 & \text { Attractor moments } & 7\end{array}$

$\begin{array}{llr}4.2 & \text { Pseudo-thermalization time } & 9\end{array}$

$\begin{array}{lll}4.3 & \text { Comparison with Navier-Stokes, vHydro, and aHydro } & 10\end{array}$

5 Conclusions 12

\section{Introduction}

Based on theory to data comparisons produced over the course of the last decades, there is now a strong body of evidence that the dynamics of the quark-gluon plasma (QGP) created in ultrarelativistic heavy-ion collisions (URHICs) is well described by relativistic dissipative hydrodynamics [1-5]. Since the phenomenologically extracted values of the shear viscosity to entropy density ratio are finite, this implies that at early times after the nuclear pass through $(0.01-0.1 \lesssim \tau \lesssim 1 \mathrm{fm} / \mathrm{c})$, the QGP possesses large non-equilibrium corrections, e.g. large pressure anisotropy in the local rest frame. Because the system experiences large deviations from local thermal equilibrium, one might expect dissipative hydrodynamics approaches to fail at early times. In practice, however, one finds that dissipative hydrodynamics describes the evolution of the components of the energy-momentum tensor quite well after a rather short time scale $\tau_{\text {hydro }} \sim 0.5-1 \mathrm{fm} / \mathrm{c}$ in the center of the overlap region for a central collision. Since dissipative hydrodynamics frameworks perform well after $\tau \sim \tau_{\text {hydro }}$, the system is said to hydrodynamize at this time scale [6]. The time scale for hydrodynamization has been extracted by comparing numerical solutions of the underlying microscopic dynamical equations to dissipative hydrodynamics evolution in both the weak and strong coupling limits [6-10]. From these studies one finds that $\tau_{\text {hydro }} \sim 2 / T$. At the highest LHC energies and assuming $\eta / s=0.2$, this translates into $\tau_{\text {hydro }} \sim 0.5 \mathrm{fm} / \mathrm{c}$ when considering the center of the fireball created in a zero impact parameter collision. 
The fact that the system is quickly driven towards dissipative hydrodynamical evolution can be understood using the concept of the hydrodynamical attractor [11]. In $0+1 \mathrm{~d}$ conformal viscous hydrodynamics, one can reduce the two coupled equations for the energy density and the shear pressure correction to a single ordinary differential equation which, subject to the correct boundary conditions, provides a universal "attractor" solution for the scaled shear correction $\bar{\pi}=\pi_{\eta}^{\eta} / \varepsilon$ as a function of the scaled time $\bar{w}=\tau / \tau_{\text {eq }}$, for example. If one solves the hydrodynamic equations with different initial conditions and plots the results versus $\bar{w}$, one finds that the solutions with different initial conditions converge to the universal attractor solution on a very short time scale (in the sense of small $\bar{w}$ ). This observation is not restricted to second-order viscous hydrodynamics and has been shown to hold in numerical solutions to Einstein's equations obtained in the strong coupling limit of $\mathcal{N}=4$ supersymmetric Yang-Mills in the large $N$ limit [7-9], QCD effective kinetic theory simulations [8, 9, 12], third-order viscous hydrodynamics [13], anisotropic hydrodynamics [10], and exact solutions to the Boltzmann equation in relaxation time approximation (RTA) subject to both Bjorken and Gubser flows [9, 10, 14-18].

Recently, using the exact solution of the RTA Boltzmann equation subject to Bjorken flow, it was demonstrated that the idea of the non-equilibrium attractor can be extended beyond the low-order moments of the one-particle distribution function typically considered in hydrodynamic approaches [17]. In ref. [17] it was demonstrated that the full one-particle distribution exhibits attractor-like behavior and that higher moments, $\mathcal{M}^{n m}$ of the oneparticle distribution function converge more quickly to their respective attractors, with the exception being moments with $m=0$, which are more sensitive to the squeezed freestreaming part of the exact solution. For moments with large $m$ and $n$, ref. [17] showed that there is a parametrically large separation between the scaled time at which solutions converge to the non-equilibrium attractor $\bar{w}_{c}$ and the time at which the moment approaches to within $10 \%$ of its equilibrium value $\bar{w}_{\text {therm }}$. Finally, In ref. [17] comparisons were made between the exact attractor moments and various dissipative hydrodynamics frameworks including relativistic Navier-Stokes (NS) [19-21], second order viscous hydrodynamics [2247], third-order viscous hydrodynamics [48, 49], and anisotropic hydrodynamics [5, 50-72]. It was found that in all cases anisotropic hydrodynamics provided the best approximation to the exact attractor irregardless of the moment considered.

Importantly, it was shown that, when $m$ or $n$ are large, both the Navier-Stokes and second order viscous hydrodynamics results for the attractor failed to describe the exact solution. The fact that a subset of the exact moment solutions converge to something that is not well-described by traditional viscous hydrodynamics treatments means that we must refine our terminology a bit: instead of calling the convergence to the attractor "hydrodynamization", we should instead call it pseudo-thermalization to emphasize that the attractor has a non-hydrodynamic nature reflected in the behavior of higher moments of the one-particle distribution function. In addition, we can associate the loss of memory of the precise initial conditions used with the pseudo-thermalization of the system. This is similar to the loss of memory which occurs if a system fully thermalizes, but with the universal state which emerges after pseudo-thermalization being far from equilibrium.

In this paper, we extend ref. [17] to study the effect of imposing number conservation on the dynamics and underlying non-equilibrium attractor. In RTA, one can enforce 
number conservation by introducing a fugacity (chemical potential) in both the dynamical and equilibrium distribution functions [73-76]. Requiring both energy and number conservation, one can derive two coupled integral equations which can be solved iteratively in order to obtain the effective temperature $T$ and fugacity $\Gamma$ as a function of proper-time. We demonstrate that for classical statistics, the integral equations can be written solely in terms of the rescaled variables $\hat{T}=T / T_{0}$ and $\hat{\Gamma}=\Gamma / \Gamma_{0}$ and the initial momentum space anisotropy $\xi_{0}$. As a result, one can construct the exact solution from any initial temperature and fugacity from a trivial scaling of the solution obtained for $\hat{T}$ and $\hat{\Gamma}$. We then determine the attractor solution to the coupled integral equations numerically by finding solutions which obey $\lim _{\tau \rightarrow 0} \mathcal{P}_{L} / \mathcal{P}_{T} \rightarrow 0$. We find that, in general, the resulting attractor solutions do not reach chemical equilibrium at late times, i.e. $\lim _{\tau \rightarrow \infty} \Gamma(\tau) \neq 1$. Despite the existence of a finite chemical potential at late times, we still observe attractor behavior in all moments and the full distribution function itself.

The structure of this paper is as follows. In section 2, we briefly review how to rewrite the $0+1 \mathrm{~d}$ RTA Boltzmann equation using boost invariant variables. In section 3 we present the integral equations obeyed by the one-particle distribution function and all moments of the one-particle distribution function. In section 4, we present our numerical results and discussion of the results. In section 5, we present our conclusions and an outlook for the future.

\section{Setup}

Our starting point is the Boltzmann equation for massless particles

$$
p^{\mu} \partial_{\mu} f(x, p)=C[f(x, p)]
$$

in RTA,

$$
C[f]=-\frac{p \cdot u}{\tau_{\mathrm{eq}}}\left(f-f_{\mathrm{eq}}\right) .
$$

The relaxation time $\tau_{\text {eq }}$ above can depend on proper time, however, since the system is conformal (massless) it must be proportional to the inverse effective temperature. The equilibrium distribution function $f_{\text {eq }}$ may be taken to be a Bose-Einstein, Fermi-Dirac, or Boltzmann distribution. Here we will assume that $f_{\text {eq }}$ is given by a Boltzmann distribution

$$
f_{\text {eq }}(\tau, p)=\Gamma(\tau) \exp \left(-\frac{p \cdot u(\tau)}{T(\tau)}\right) .
$$

where $\Gamma(\tau)=\exp \left(-\mu_{\mathrm{eff}}(\tau) / T(\tau)\right)$ is the effective fugacity with $\mu_{\mathrm{eff}}(\tau)$ and $T(\tau)$ being the local effective chemical potential and temperature, respectively.

The effective temperature $T$ and fugacity $\Gamma$ will be obtained via matching conditions which demand that the energy and number densities calculated from the dynamical distribution function $f$ be equal to the energy and number densities determined from the equilibrium distribution, $f_{\text {eq }}$. The quantity $u^{\mu}$ represents the four-velocity of the local rest frame of the matter which herein we assume to be given by the transversally homogenous and boost invariant Bjoken flow $(0+1 \mathrm{~d})$. 
In equilibrium, for massless particles obeying classical statistics the particle density, entropy density, energy density, and pressure are

$$
\begin{array}{ll}
n_{\text {eq }}=\frac{\Gamma T^{3}}{\pi^{2}}, & \mathcal{S}_{\text {eq }}=\frac{4 \Gamma T^{3}}{\pi^{2}}, \\
\mathcal{E}_{\text {eq }}=\frac{3 \Gamma T^{4}}{\pi^{2}}, & \mathcal{P}_{\text {eq }}=\frac{\Gamma T^{4}}{\pi^{2}} .
\end{array}
$$

\subsection{Boost-invariant variables}

For one-dimensional boost-invariant expansion, all scalar functions of time and space depend only on the longitudinal proper time $\tau \equiv \sqrt{t^{2}-z^{2}}$. In addition, the hydrodynamic flow $u^{\mu}$ has the following form $u^{\mu}=\left(\frac{t}{\tau}, 0,0, \frac{z}{\tau}\right)$ [77]. One can introduce a space-like vector that is orthogonal in all frames and corresponds to the z-direction in the local rest frame of the matter $z^{\mu}=\left(\frac{z}{\tau}, 0,0, \frac{t}{\tau}\right)$. The requirement of boost invariance implies that $f(x, p)$ can depend only on three variables: $\tau, w$ and $\vec{p}_{T}$ [78-81]. The boost-invariant variable $w$ is defined by

$$
w \equiv t p_{z}-z E,
$$

where $z$ is the spatial coordinate, not to be confused with the basis vector $z^{\mu}$. Using $w$ and $\vec{p}_{T}$ one can define

$$
v \equiv E t-p_{z} z=\sqrt{w^{2}+\left(m^{2}+\vec{p}_{T}^{2}\right) \tau^{2}} .
$$

From (2.5) and (2.6) one can easily find the energy and the longitudinal momentum of a particle

$$
E=p^{0}=\frac{v t+w z}{\tau^{2}}, \quad p_{z}=\frac{w t+v z}{\tau^{2}} .
$$

The momentum integration measure in phase-space is

$$
d P=\frac{d^{4} p}{(2 \pi)^{4}} 2 \pi \delta\left(p^{2}-m^{2}\right) 2 \theta\left(p^{0}\right)=\frac{d p_{z}}{(2 \pi)^{3} p^{0}} d^{2} p_{T}=\frac{d w d^{2} p_{T}}{(2 \pi)^{3} v} .
$$

In the following we shall consider massless partons, $m=0$.

\subsection{Boost-invariant form of the kinetic equation}

Making use of the boost-invariant variables introduced in the previous subsection, one finds $p^{\mu} \partial_{\mu} f=\frac{v}{\tau} \frac{\partial f}{\partial \tau}, p \cdot u=\frac{v}{\tau}$, and $p \cdot z=-\frac{w}{\tau}$. With this, eq. (2.1) becomes simply [80, 81]

$$
\frac{\partial f\left(\tau, w, p_{T}\right)}{\partial \tau}=\frac{f_{\mathrm{eq}}\left(\tau, w, p_{T}\right)-f\left(\tau, w, p_{T}\right)}{\tau_{\mathrm{eq}}(\tau)},
$$

with the finite chemical potential equilibrium distribution function (2.3) given by

$$
f_{\mathrm{eq}}\left(\tau, w, p_{T}\right)=\Gamma(\tau) \exp \left[-\frac{\sqrt{w^{2}+p_{T}^{2} \tau^{2}}}{T(\tau) \tau}\right] .
$$

Note also that $f\left(\tau, w, \vec{p}_{T}\right)$ is an even function of $w$ and depends only on the magnitude of the transverse momentum $\vec{p}_{T}$. 


\section{Exact solution for the distribution function}

The formal solution of the kinetic equation $(2.9)$ is $[80,81]$

$$
f\left(\tau, w, p_{T}\right)=D\left(\tau, \tau_{0}\right) f_{0}\left(w, p_{T}\right)+\int_{\tau_{0}}^{\tau} \frac{d \tau^{\prime}}{\tau_{\mathrm{eq}}\left(\tau^{\prime}\right)} D\left(\tau, \tau^{\prime}\right) f_{\mathrm{eq}}\left(\tau^{\prime}, w, p_{T}\right),
$$

where we have introduced the damping function

$$
D\left(\tau_{2}, \tau_{1}\right)=\exp \left[-\int_{\tau_{1}}^{\tau_{2}} \frac{d \tau^{\prime \prime}}{\tau_{\text {eq }}\left(\tau^{\prime \prime}\right)}\right] .
$$

For $\tau=\tau_{0}$ the distribution function $f$ reduces to the initial distribution function, $f_{0}$. For the conformal RTA solution we use the relation

$$
\tau_{\mathrm{eq}}(\tau)=\frac{5 \bar{\eta}}{T(\tau)},
$$

where $\bar{\eta} \equiv \eta / \mathcal{S}$ is the ratio of the shear viscosity $\eta$ to entropy density $\mathcal{S}$.

\subsection{Initial distribution}

Herein, for the initial condition we take the Romatschke-Strickland form [82] with a classical Boltzmann distribution as the underlying isotropic distribution

$$
f_{0}\left(w, p_{T}\right)=\gamma_{0} \exp \left[-\frac{\sqrt{\left(1+\xi_{0}\right) w^{2}+p_{T}^{2} \tau_{0}^{2}}}{\Lambda_{0} \tau_{0}}\right]=\gamma_{0} \exp \left[-\frac{\sqrt{w^{2} \alpha_{0}^{-2}+p_{T}^{2} \tau_{0}^{2}}}{\Lambda_{0} \tau_{0}}\right],
$$

where, in the second equality, we have introduced the elliptical anisotropy parameter $\alpha \equiv 1 / \sqrt{1+\xi}$ for convenience. The distribution function above reduces to an isotropic Boltzmann distribution if the anisotropy parameter $\alpha_{0}=\alpha\left(\tau_{0}\right)=1$. If $\alpha_{0}=1$, the transverse momentum scale $\Lambda_{0}$ is equal to the system's initial temperature $T_{0}$ and the initial microscopic fugacity $\gamma_{0}$ is equal to the initial effective fugacity $\Gamma_{0}$. In general, one must use Landau matching of the initial energy and number densities to fix $\Lambda_{0}$ and $\gamma_{0}$ in terms of $T_{0}$ and $\Gamma_{0}$. The resulting "matching conditions" are [76]

$$
\begin{aligned}
T & =\frac{\mathcal{H}(\alpha)}{2 \alpha} \Lambda, \\
\Gamma & =\frac{8 \gamma \alpha^{4}}{\mathcal{H}^{3}(\alpha)},
\end{aligned}
$$

where $\mathcal{H}(\alpha) \equiv \mathcal{H}^{20}(\alpha)$. The special functions $\mathcal{H}^{n m}$ needed are

$$
\mathcal{H}^{n m}(y) \equiv \frac{2 y^{2 m+1}}{2 m+1}{ }_{2} F_{1}\left(\frac{1}{2}+m, \frac{1-n}{2} ; \frac{3}{2}+m ; 1-y^{2}\right) .
$$

\subsection{General moments of the distribution function}

In order to solve eq. (3.1), one can take a general moment of both sides using ${ }^{1}$

$$
\mathcal{M}^{n m}[f] \equiv \int d P(p \cdot u)^{n}(p \cdot z)^{2 m} f\left(\tau, w, p_{T}\right)
$$

\footnotetext{
${ }^{1}$ For the boost-invariant case considered herein the exact distribution function is symmetric under $p \cdot z \rightarrow$ $-p \cdot z$. As a result, all moments with odd powers of $p \cdot z$ are zero. We, therefore, only consider moments with even powers of $p \cdot z$.
} 
For $n=2$ and $m=0$, one obtains the energy density

$$
\mathcal{E}=\mathcal{M}^{20}=\int d P(p \cdot u)^{2} f\left(\tau, w, p_{T}\right)=T_{\mathrm{LRF}}^{00},
$$

and, for $n=1$ and $m=0$, one obtains the number density

$$
n=\mathcal{M}^{10}=\int d P(p \cdot u) f\left(\tau, w, p_{T}\right),
$$

Using the mass shell constraint, one can always rewrite the transverse momentum squared in terms of the energy and longitudinal momentum, so that any moment containing $p_{T}^{2 \ell}$ can be written as a linear combination of the $\mathcal{M}^{n m}$ moments above. As a result, in the general case, we need to compute

$$
\mathcal{M}^{n m}[f]=\frac{1}{(2 \pi)^{3} \tau^{n+2 m}} \int d w d^{2} p_{T} v^{n-1} w^{2 m} f\left(\tau, w, p_{T}\right)
$$

\subsection{Integral equation obeyed by a general moment}

Taking a general moment of eq. (3.1) one obtains

$$
\mathcal{M}^{n m}(\tau)=D\left(\tau, \tau_{0}\right) \mathcal{M}_{0}^{n m}(\tau)+\int_{\tau_{0}}^{\tau} \frac{d \tau^{\prime}}{\tau_{\mathrm{eq}}\left(\tau^{\prime}\right)} D\left(\tau, \tau^{\prime}\right) \mathcal{M}_{\mathrm{eq}}^{n m}\left(\tau, \tau^{\prime}\right)
$$

where

$$
\begin{aligned}
\mathcal{M}_{0}^{n m}(\tau) & =\frac{\gamma_{0}(n+2 m+1) ! \Lambda_{0}^{n+2 m+2}}{(2 \pi)^{2}} \mathcal{H}^{n m}\left(\frac{\alpha_{0} \tau_{0}}{\tau}\right), \\
\mathcal{M}_{\mathrm{eq}}^{n m}\left(\tau, \tau^{\prime}\right) & =\frac{\Gamma\left(\tau^{\prime}\right)(n+2 m+1) ! T^{n+2 m+2}\left(\tau^{\prime}\right)}{(2 \pi)^{2}} \mathcal{H}^{n m}\left(\frac{\tau^{\prime}}{\tau}\right) .
\end{aligned}
$$

One can rewrite the first term, which involves the initial values of the microscopic parameters $\gamma_{0}$ and $\Lambda_{0}$, in terms of the initial effective fugacity $\Gamma_{0}$ and temperature $T_{0}$ using eqs. (3.5) and (3.6). Putting the pieces together, our final result for the general moment equation is

$$
\begin{aligned}
\mathcal{M}^{n m}(\tau)=\frac{(n+2 m+1) !}{(2 \pi)^{2}}\left[D\left(\tau, \tau_{0}\right) \alpha_{0}^{n+2 m-2} T_{0}^{n+2 m+2} \Gamma_{0} \frac{\mathcal{H}^{n m}\left(\frac{\alpha_{0} \tau_{0}}{\tau}\right)}{\left[\mathcal{H}\left(\alpha_{0}\right) / 2\right]^{n+2 m-1}}\right. & \\
& \left.\quad+\int_{\tau_{0}}^{\tau} \frac{d \tau^{\prime}}{\tau_{\mathrm{eq}}\left(\tau^{\prime}\right)} D\left(\tau, \tau^{\prime}\right) \Gamma\left(\tau^{\prime}\right) T^{n+2 m+2}\left(\tau^{\prime}\right) \mathcal{H}^{n m}\left(\frac{\tau^{\prime}}{\tau}\right)\right] .
\end{aligned}
$$

Final equations. From eq. (3.15) we can obtain two integral equations by evaluating the $n=1$ and $m=0$ and $n=2$ and $m=0$ moments which map to the number density and energy density, respectively, with the results being

$$
\Gamma(\tau) T^{4}(\tau)=D\left(\tau, \tau_{0}\right) \Gamma_{0} T_{0}^{4} \frac{\mathcal{H}\left(\frac{\alpha_{0} \tau_{0}}{\tau}\right)}{\mathcal{H}\left(\alpha_{0}\right)}+\int_{\tau_{0}}^{\tau} \frac{d \tau^{\prime}}{2 \tau_{\mathrm{eq}}\left(\tau^{\prime}\right)} D\left(\tau, \tau^{\prime}\right) \Gamma\left(\tau^{\prime}\right) T^{4}\left(\tau^{\prime}\right) \mathcal{H}\left(\frac{\tau^{\prime}}{\tau}\right),
$$

and

$$
\Gamma(\tau) T^{3}(\tau)=\frac{1}{\tau}\left[D\left(\tau, \tau_{0}\right) \Gamma_{0} T_{0}^{3} \tau_{0}+\int_{\tau_{0}}^{\tau} \frac{d \tau^{\prime}}{\tau_{\mathrm{eq}}\left(\tau^{\prime}\right)} D\left(\tau, \tau^{\prime}\right) \Gamma\left(\tau^{\prime}\right) T^{3}\left(\tau^{\prime}\right) \tau^{\prime}\right]
$$


where we used the matching conditions $\mathcal{E}=\mathcal{E}_{\text {eq }}(T, \Gamma)$ and $n=n_{\text {eq }}(T, \Gamma)$ on the lefthand-side and the fact that $\mathcal{H}^{10}(\alpha)=2 \alpha$ to simplify the second integral equation. Note, importantly, that one can divide the left- and right-hand sides of eqs. (3.16) and (3.17) by the initial fugacity $\Gamma_{0}$ and rewrite them entirely in terms of $\hat{\Gamma} \equiv \Gamma / \Gamma_{0}$. As a result, one can solve these coupled integral equations with a given value of $\Gamma_{0}$, e.g. $\Gamma_{0}=1$, and then obtain solutions with different initial fugacity by scaling the result by the initial fugacity. ${ }^{2}$

\section{Results}

For our results, we solve eqs. (3.16) and (3.17) numerically to obtain $T(\tau)$ and $\Gamma(\tau)$ given a set of initial values at $\tau_{0}: T_{0}, \Gamma_{0}$, and $\alpha_{0}$. For this purpose we wrote a CUDA-based GPU code which allows one to efficiently solve eqs. (3.16) and (3.17) on very large propertime lattices using a logarithmically-spaced grid. For all results presented herein we used a temporal lattice size of 4096 points and iterated the coupled integral equations until the effective temperature and fugacity converged to sixteen digits at all proper times. The code used to produce our results is included in the arXiv bundle for this paper and is also publicly available for download using the link provided in ref. [83]. Once the solutions for the effective temperature and fugacity are obtained, one can use eq. (3.15) to obtain the proper-time dependence of any moment required. One can also use eq. (3.1) to reconstruct the full one-particle distribution in a grid in momentum-space.

We will present the resulting exact solutions for the scaled moments

$$
\overline{\mathcal{M}}^{n m}(\tau) \equiv \frac{\mathcal{M}^{n m}(\tau)}{\mathcal{M}_{\mathrm{eq}}^{n m}(\tau)},
$$

where

$$
\mathcal{M}_{\mathrm{eq}}^{n m}(\tau)=\mathcal{M}_{\mathrm{eq}}^{n m}(\tau, \tau)=\frac{(n+2 m+1) ! \Gamma(\tau) T^{n+2 m+2}(\tau)}{2 \pi^{2}(2 m+1)},
$$

are the moments associated with an equilibrium Boltzmann distribution function. The scaled moments approach one at late times by construction and the rate at which they approach one provides a quantitative measure of how quickly the system thermalizes. Note that higher moments are sensitive to higher average momenta where the hydrodynamics assumption of small gradients could fail.

\subsection{Attractor moments}

In figure 1, we present sixteen panels containing our numerical results for the scaled moments $\overline{\mathcal{M}}^{n m}(\tau)$ with $m, n \in\{0,1,2,3\}$. In all panels, the horizontal axis of figure 1 is the scaled proper-time $\bar{w} \equiv \tau / \tau_{\text {eq }}=\tau T / 5 \bar{\eta}$, the black line is the exact solution for the attractor, and the various dashed/dotted curves are exact solutions with different values of $\alpha_{0}$. To obtain the attractor solution, we solved the coupled integral equations (3.16) and (3.17) with $\tau_{0}=10^{-3} \mathrm{fm} / \mathrm{c}, T_{0}=1 \mathrm{GeV}, \alpha_{0}=2.5 \times 10^{-2}$, and $\Gamma_{0}=1$. To obtain the specific solutions

\footnotetext{
${ }^{2} \mathrm{~A}$ similar scaling can be done with $T_{0}$, however, in addition to scaling eqs. (3.16) and (3.17) by $T_{0}^{4}$ and $T_{0}^{3}$, respectively, one must also make a change of variables in the proper-time integrations by introducing $\hat{\tau} \equiv \tau T_{0}$.
} 

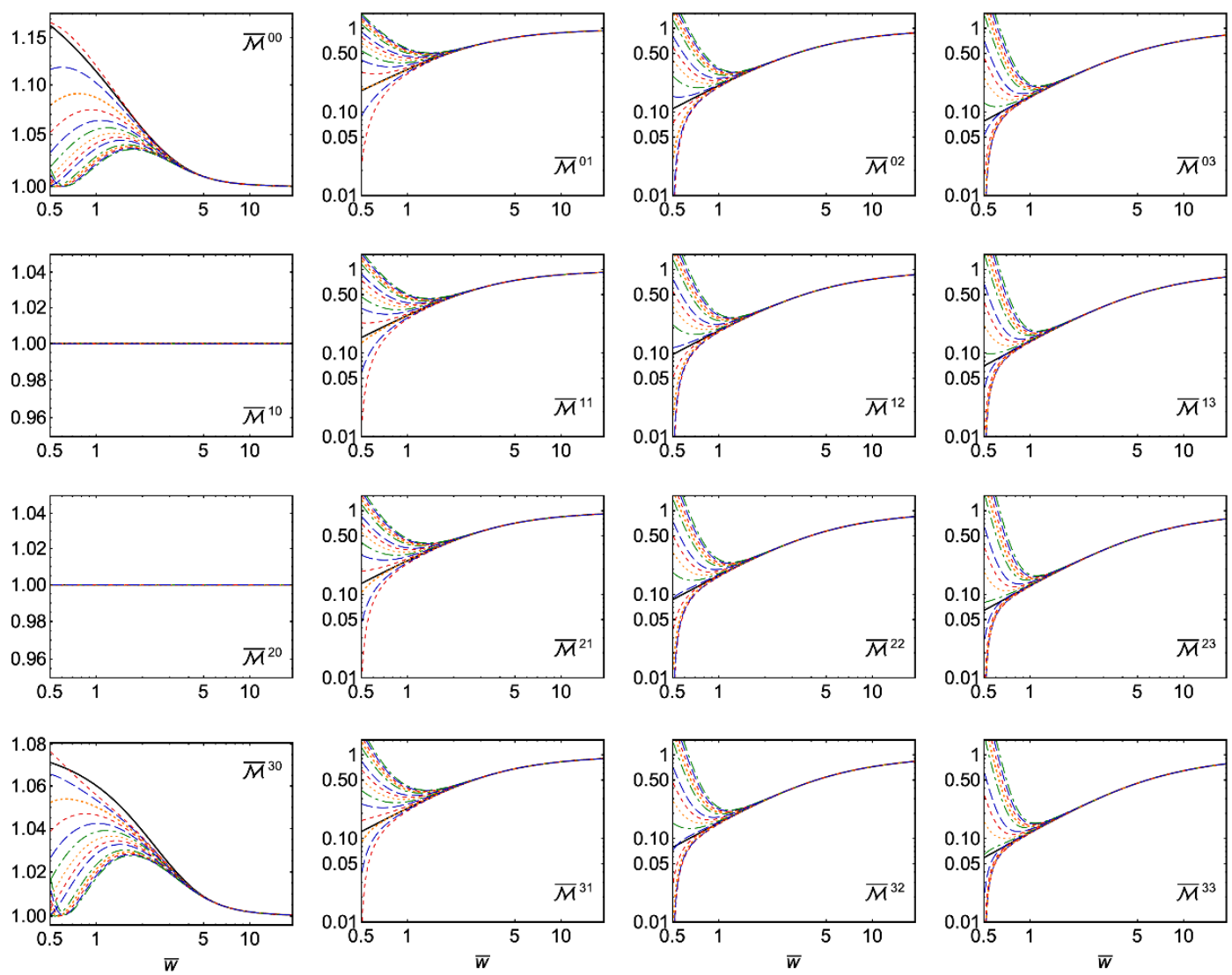

Figure 1. The scaled moments $\overline{\mathcal{M}}^{n m}=\mathcal{M}^{n m}(\tau) / \mathcal{M}_{\text {eq }}^{n m}(\tau)$ obtained from the exact attractor solution (solid black line) are compared to a set of exact solutions (various colored dotted and dashed lines) initialized with varying $\alpha_{0}$. The horizontal axis is $\bar{w} \equiv \tau / \tau_{\text {eq }}=\tau T / 5 \bar{\eta}$. Panels show a grid in $n$ and $m$.

(dashed/dotted lines), we solved the same coupled integral equations (3.16) and (3.17) with $\tau_{0}=10^{-1} \mathrm{fm} / \mathrm{c}, T_{0}=1 \mathrm{GeV}, \alpha_{0} \in 0.1 \ldots 1.5$, and $\Gamma_{0}=1$. The range of initial anisotropies considered covers both very oblate and very prolate initial momentum-space anisotropy.

As can be seen from this figure, for generic initial conditions, all moments with $m>0$ visually converge to their respective attractors after a short rescaled time $\bar{w} \sim 2$. For $\overline{\mathcal{M}}^{10}$ (scaled number density) and $\overline{\mathcal{M}}^{20}$ (scaled energy density), we see that the constraints are properly enforced, resulting in these moments being equal to their equilibrium values at all proper times. For moments with $m=0$, we see a somewhat slower approach to the attractor. This is similar to what was found when not enforcing number conservation [17], however, herein we see smaller deviations from equilibrium. Despite these smaller deviations from equilibrium compared to the prior studies, moments with $m=0$ still converge more slowly than other moments. The slow convergence of moments with $m=0$ is related to the fact that they contain no powers of $p_{z}$ in their integrands and are, therefore, more sensitive to the free streaming term (first term) in eq. (3.1). Free streaming results in momentum modes from the initial distribution being squeezed to smaller and smaller $\left|p_{z}\right|$ as a function of proper time (see ref. [17] for details). 

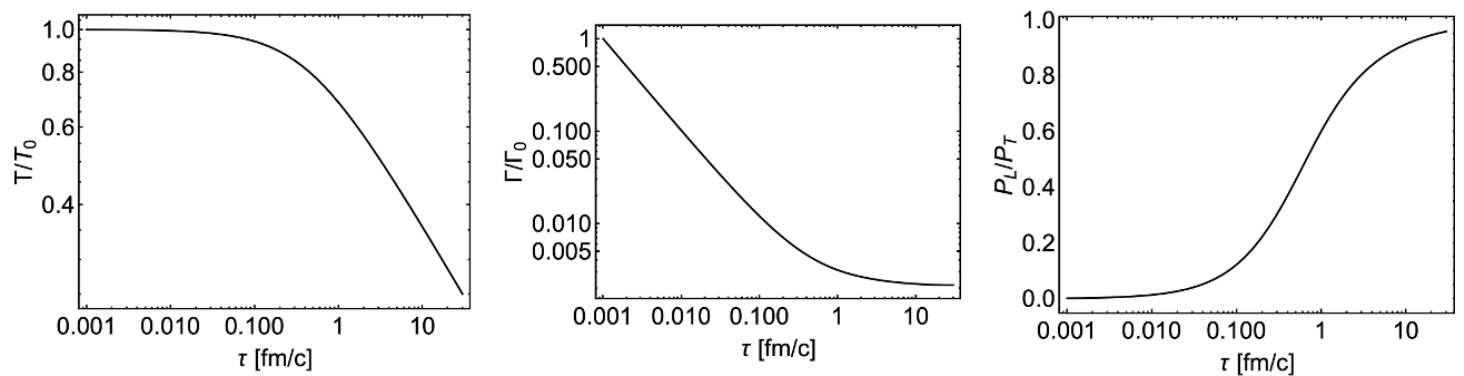

Figure 2. Three panels showing (left) the scaled effective temperature, (middle) the scaled fugacity, and (right) the pressure anisotropy as a function of time for "attractor" initial conditions $\tau_{0}=10^{-3} \mathrm{fm} / \mathrm{c}, T_{0}=1 \mathrm{GeV}, \alpha_{0}=2.5 \times 10^{-2}$, and $\Gamma_{0}=1$.

Note, however, that although all scaled moments approach one in the large $\bar{w}$ limit, the system generically possesses a finite fugacity at late times. ${ }^{3}$ To demonstrate this, we plot the effective temperature, effective fugacity, and the pressure anisotropy associated with the attractor in figure 2. As can be seen from this figure, the scaled temperature evolution (left panel) obtained using attractor initial conditions shows characteristics of early time free streaming, for which the temperature scale (average momentum scale) is constant $[51,84-$ 86], followed by a power law decrease at late time indicative of hydrodynamic evolution. The attractor's scaled effective fugacity (middle panel) decreases as a power law at early times and eventually saturates at late times. Finally, we see that attractor's pressure anisotropy is large (highly oblate) at early times with $P_{L} \ll P_{T}$ and then slowly relaxes towards isotropy at late times.

\subsection{Pseudo-thermalization time}

In order to quantitatively assess the convergence of generic exact solutions to the attractor for each moment, one can compute the scaled time at which all solutions collapse to the attractor by requiring that $\max \left|\overline{\mathcal{M}}_{\mathrm{i}}^{n m}\left(\bar{w}_{c}\right)-\overline{\mathcal{M}}_{\text {attractor }}^{n m}\left(\bar{w}_{c}\right)\right|<\delta_{c}$ for $i$ in the entire set of trial runs. In ref. [17], $\delta_{c}=10^{-6}$ was chosen in order to require that the solutions were extremely well converged to the attractor. Herein, we will also consider the weaker convergence criteria of $\delta_{c}=10^{-2}$, which should correspond more closely to the time that one extracts when visually checking for convergence in figure 1 .

In figures 3 and 4 we plot the convergence or pseudo-thermalization time $\bar{w}_{c}$ with $\delta_{c}=10^{-6}$ and $\delta_{c}=10^{-2}$, respectively. Figure 3 uses the strong convergence criteria of $\delta_{c}=10^{-6}$ which was the condition used in ref. [17]. The two top panels show $\bar{w}_{c}$ as a function of $m$ and $n$ and the bottom panel shows the case $m=0$ as a function of $n$. As can be seen from the top left panel, for $m \geq 2, \bar{w}_{c}$ is a decreasing function of $m$ and $n$. From the top right panel we see that for $m=1$ the pseudo-thermalization time increases at large $n$, but moments with $m>1$ have a pseudo-thermalization which decreases as $n$ increases. Turning to the bottom panel $(m=0)$, we see that the $n=1$ and $n=2$ moments thermalize "instantly" since these are enforced by conservation laws ${ }^{4}$ and we see a strong increase in $\bar{w}_{c}$

\footnotetext{
${ }^{3}$ One can adjust the late-time fugacity by changing the initial fugacity $\Gamma_{0}$.

${ }^{4}$ In this case we set $\bar{w}_{c}$ to be the smallest scaled time in the temporal grid.
} 

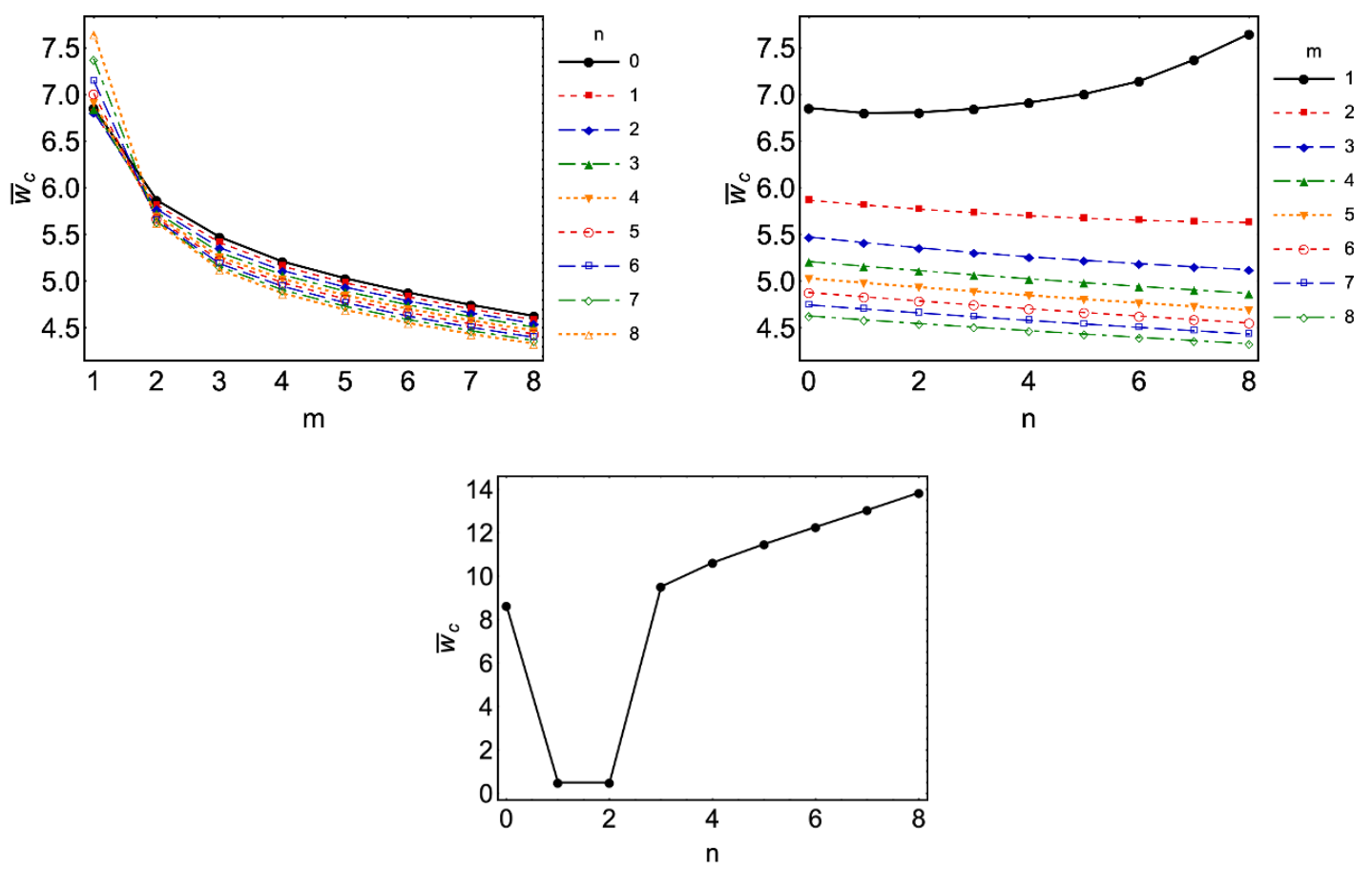

Figure 3. The pseudo-thermalization time $\bar{w}_{c}$ for $n, m \in\{0, \cdots, 8\}$ and $\delta_{c}=10^{-6}$. The top left panel shows $\bar{w}_{c}$ as a function of $m$ with the lines corresponding to different values of $n$. The top right panel shows $\bar{w}_{c}$ as a function of $n$ with the lines corresponding to different values of $m$. The bottom panel shows $\bar{w}_{c}$ as a function of $n$ for the case $m=0$.

as $n$ increases. In the range of $n$ and $m$ shown, the maximum pseudo-thermalization time is $\bar{w}_{c}^{\max } \simeq 14$. This can be compared with the maximum pseudo-thermalization time obtained without enforcing number conservation (see figure 8 in ref. [17]), which was $\bar{w}_{c}^{\max } \simeq 28$.

Turning to figure 4 we see the same three panels but now for the weaker convergence criterium of $\delta_{c}=0.01$. As can be seen from these figures, one obtains a shorter pseudothermalization time with the weaker convergence criteria, as expected. In addition, the scaled times extracted for moments with $m \neq 0$ are in the range of $\bar{w}_{c} \sim 1-3$. For a RHIC energy heavy-ion collision with a typical initial central temperature of $500 \mathrm{MeV}$ at $\tau_{0}=0.1 \mathrm{fm} / \mathrm{c}$ this translates into a physical pseudo-thermalization time of $\tau_{c} \sim 0.5-3 \mathrm{fm} / \mathrm{c}$ with the precise value depending on the mode considered. We emphasize that the higher $n$ and $m$ moments converge more quickly and have pseudo-thermalization times on the low side of this window, while the lower $n$ and $m$ moments converge more slowly to the attractor.

\subsection{Comparison with Navier-Stokes, vHydro, and aHydro}

Finally, we compare the exact results for the scaled attractors moments with results obtained from anisotropic hydrodynamics (aHydro) and second-order viscous hydrodynamics (vHydro). For vHydro, we use the complete second-order viscous hydrodynamics equations of Denicol, Niemi, Molnar, and Rischke (DNMR) [40, 87]. For aHydro, we use the moments method introduced originally by Florkowski and Tinti [57]. For both vHydro 

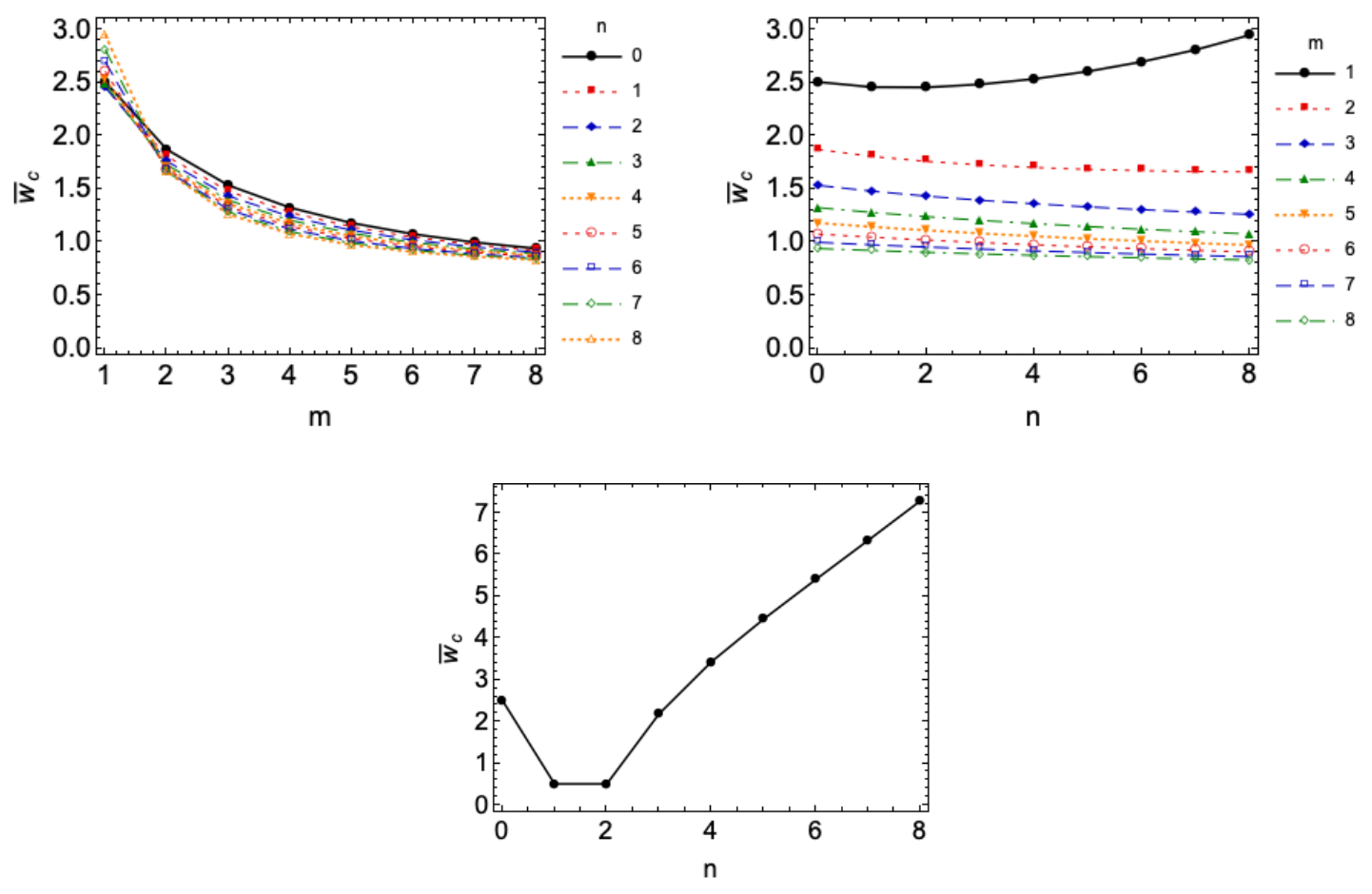

Figure 4. The pseudo-thermalization time $\bar{w}_{c}$ for $n, m \in\{0, \cdots, 8\}$ and $\delta_{c}=10^{-2}$. Panels are the same as in figure 3.

and aHydro, the attractor is determined from the solution of a one-dimensional ordinary differential equation subject to the appropriate initial condition. For details concerning the determination of the attractor for both aHydro and vHydro, we refer to the reader to ref. [10].

For vHydro, one extracts $\bar{\pi}=\pi / \epsilon$ and, using this, one can reconstruct the solution for any moment using [17]

$$
\overline{\mathcal{M}}_{\mathrm{vHydro}}^{n m}(\tau)=1-\frac{3 m(n+2 m+2)(n+2 m+3)}{4(2 m+3)} \bar{\pi} .
$$

One can obtain the Navier-Stokes (NS) result by taking $\bar{\pi}=16 \bar{\eta} /(9 \tau T)$ in (4.3).

For aHydro, one extracts the anisotropy parameter $\alpha(\tau)$ associated with the attractor solution. Once this is determined one can use eq. (4.4) of ref. [17], modified to take into account finite fugacity, to obtain a compact expression for any moment

$$
\overline{\mathcal{M}}_{\mathrm{aHydro}}^{n m}(\tau)=(2 m+1)(2 \alpha)^{n+2 m-2} \frac{\mathcal{H}^{n m}(\alpha)}{[\mathcal{H}(\alpha)]^{n+2 m-1}} .
$$

The aHydro dynamical equations taking into account number conservation can be found in ref. [76]. In figure 5, I compare the exact attractor (black solid lines) with the aHydro attractor (red dashed lines), DNMR attractor (blue long dashed lines), and the NS limit (green dot-dashed lines) for each moment. In all cases shown, aHydro provides a better approximation to the exact moments than the vHydro or NS frameworks. This is similar 

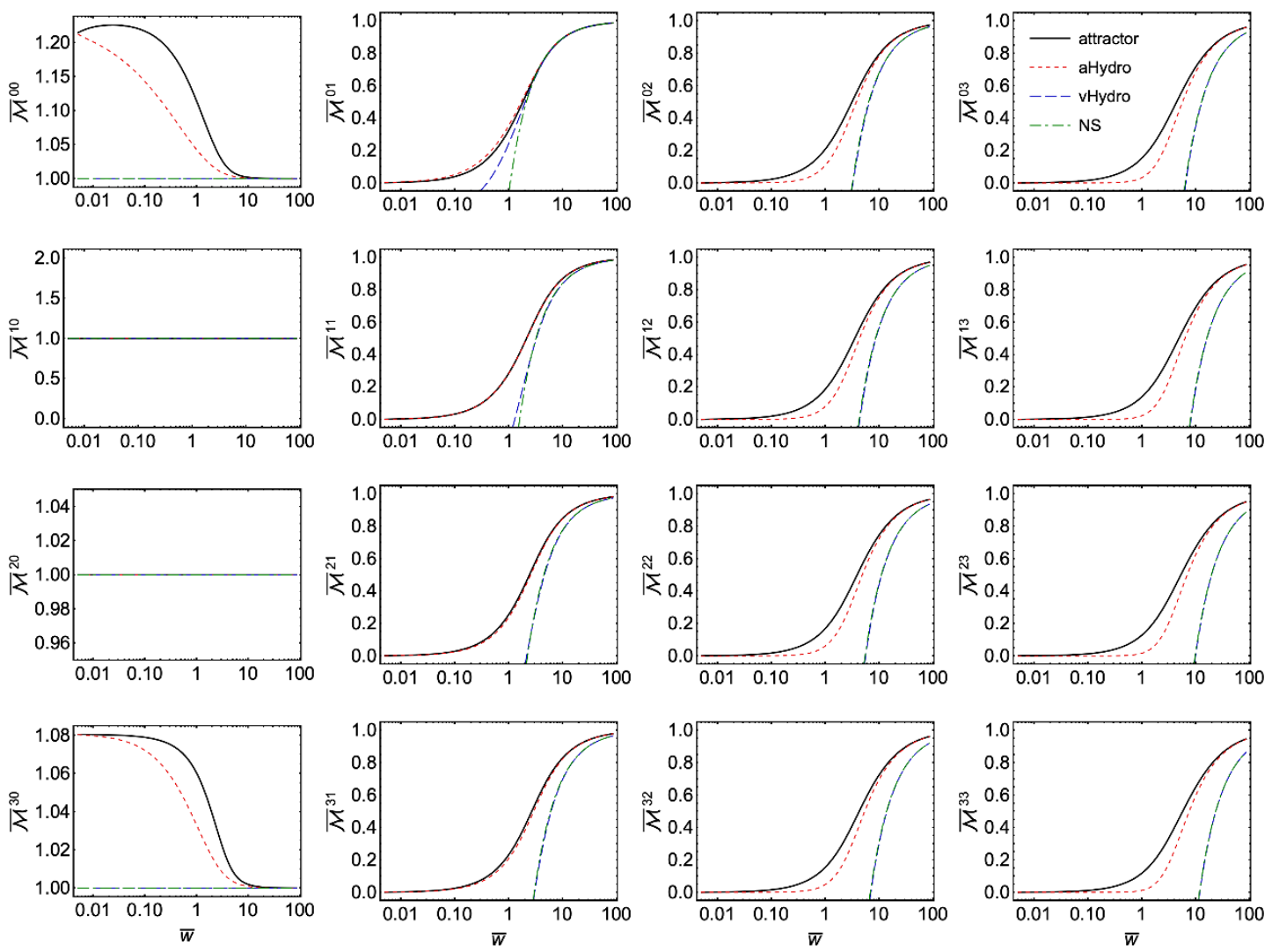

Figure 5. Scaled moments $\overline{\mathcal{M}}^{n m}$ obtained from the exact attractor solution (solid black line) compared with the aHydro attractor (red dashed lines), DNMR attractor (blue long dashed lines), and the Navier-Stokes limit for each moment (green dot-dashed lines). Horizontal axis is $\bar{w} \equiv \tau T / 5 \bar{\eta}$. Panels show a grid in $n$ and $m$.

to what was found in the case where number conservation was not imposed [17]. Finally, we note that, for aHydro, the moment with the best agreement is the $n=1$ and $m=1$ moment, for which the two results are virtually indistinguishable. This can be contrasted with ref. [17] which found that it was the $n=0$ and $m=1$ moment which was best described when number conservation was not imposed. It is not clear to us why this would be the case.

\section{Conclusions}

In this paper we extended previous studies of the conformal $0+1 \mathrm{~d}$ kinetic non-equilibrium attractor in relaxation time approximation by imposing number conservation through the introduction of a dynamical fugacity (chemical potential). We derived two coupled integral equations for the effective temperature and fugacity which were then solved numerically to obtain the exact solution. We demonstrated that the resulting solutions exhibited convergence to a unique non-equilibrium attractor even though the system is out of chemical equilibrium generically $\left(\lim _{\tau \rightarrow \infty} \Gamma(\tau) \neq 1\right)$. We found that, compared to the case where number conservation was not imposed, the moments converge to their respective attractors 
more quickly. Overall, however, we found that the behavior in the two cases is qualitatively very similar, providing further evidence that the non-equilibrium attractor is ubiquitous. We also compared the resulting attractor moments with predictions of different hydrodynamic frameworks. We found that anisotropic hydrodynamics provided the best approximation to the exact results for all moments.

Looking forward, herein we used a RTA collisional kernel and enforced number conservation by introducing a dynamical fugacity. It would be interesting to look at leading-order scalar field theory, in which case one only has $2 \leftrightarrow 2$ collisions and hence a theory which automatically conserves number. Such comparisons have been made in the context of aHydro in ref. [76] where the authors studied both number-conserving RTA and scalar collisional kernels. Therein, it was shown that one could numerically extract the aHydro attractor for both RTA and scalar kernels, with the two being qualitatively similar. It would be very interesting to consider the $2 \leftrightarrow 2$ scalar kinetic theory using Monte-Carlo-based transport in order to compare with the exact results obtained herein using number-conserving RTA. It would also be interesting to make comparisons with the attractor extracted from the effective kinetic theory framework of Kurkela et al., particularly in the case that baryon number conservation is at play $[8,12,88,89]$.

\section{Acknowledgments}

We thank D. Almaalol for comments and suggestions. M.S. and U.T. were supported by the U.S. Department of Energy, Office of Science, Office of Nuclear Physics under Award No. DE-SC0013470.

Open Access. This article is distributed under the terms of the Creative Commons Attribution License (CC-BY 4.0), which permits any use, distribution and reproduction in any medium, provided the original author(s) and source are credited.

\section{References}

[1] R. Averbeck, J.W. Harris and B. Schenke, Heavy-ion physics at the LHC, in The large hadron collider: harvest of run 1, T. Schörner-Sadenius ed., Springer, U.S.A. (2015).

[2] S. Jeon and U. Heinz, Introduction to hydrodynamics, in Quark-gluon plasma 5, X.N. Wang ed., World Scientific, Singapore (2016).

[3] P. Romatschke and U. Romatschke, Relativistic fluid dynamics in and out of equilibrium, arXiv: 1712.05815 [INSPIRE].

[4] W. Florkowski, M.P. Heller and M. Spalinski, New theories of relativistic hydrodynamics in the LHC era, Rept. Prog. Phys. 81 (2018) 046001 [arXiv: 1707.02282] [InSPIRE].

[5] M. Alqahtani, M. Nopoush and M. Strickland, Relativistic anisotropic hydrodynamics, Prog. Part. Nucl. Phys. 101 (2018) 204 [arXiv:1712.03282] [INSPIRE].

[6] P.M. Chesler and L.G. Yaffe, Horizon formation and far-from-equilibrium isotropization in supersymmetric Yang-Mills plasma, Phys. Rev. Lett. 102 (2009) 211601 [arXiv:0812.2053] [INSPIRE]. 
[7] M.P. Heller, D. Mateos, W. van der Schee and M. Triana, Holographic isotropization linearized, JHEP 09 (2013) 026 [arXiv:1304.5172] [INSPIRE].

[8] L. Keegan et al., Weak and strong coupling equilibration in nonabelian gauge theories, JHEP 04 (2016) 031 [arXiv: 1512.05347] [INSPIRE].

[9] P. Romatschke, Relativistic fluid dynamics far from local equilibrium, Phys. Rev. Lett. 120 (2018) 012301 [arXiv:1704.08699] [INSPIRE].

[10] M. Strickland, J. Noronha and G. Denicol, Anisotropic nonequilibrium hydrodynamic attractor, Phys. Rev. D 97 (2018) 036020 [arXiv:1709.06644] [INSPIRE].

[11] M.P. Heller and M. Spalinski, Hydrodynamics beyond the gradient expansion: resurgence and resummation, Phys. Rev. Lett. 115 (2015) 072501 [arXiv:1503. 07514] [INSPIRE].

[12] A. Kurkela and Y. Zhu, Isotropization and hydrodynamization in weakly coupled heavy-ion collisions, Phys. Rev. Lett. 115 (2015) 182301 [arXiv: 1506.06647] [INSPIRE].

[13] C. Chattopadhyay, A. Jaiswal, S. Jaiswal and S. Pal, Analytical solutions and attractors of higher-order viscous hydrodynamics for Bjorken flow, forthcoming (2019).

[14] A. Behtash, C.N. Cruz-Camacho and M. Martinez, Far-from-equilibrium attractors and nonlinear dynamical systems approach to the Gubser flow, Phys. Rev. D 97 (2018) 044041 [arXiv: 1711.01745] [INSPIRE].

[15] A. Behtash, C.N. Cruz-Camacho, S. Kamata and M. Martinez, Non-perturbative rheological behavior of a far-from-equilibrium expanding plasma, Phys. Lett. B 797 (2019) 134914 [arXiv: 1805.07881] [INSPIRE].

[16] G.S. Denicol and J. Noronha, Hydrodynamic attractor and the fate of perturbative expansions in Gubser flow, Phys. Rev. D 99 (2019) 116004 [arXiv: 1804.04771] [INSPIRE].

[17] M. Strickland, The non-equilibrium attractor for kinetic theory in relaxation time approximation, JHEP 12 (2018) 128 [arXiv: 1809.01200] [INSPIRE].

[18] A. Behtash, S. Kamata, M. Martinez and H. Shi, Dynamical systems and nonlinear transient rheology of the far-from-equilibrium Bjorken flow, Phys. Rev. D 99 (2019) 116012 [arXiv: 1901.08632] [INSPIRE].

[19] C. Eckart, The thermodynamics of irreversible processes. 3. Relativistic theory of the simple fluid, Phys. Rev. 58 (1940) 919 [InSPIRE].

[20] L.D. Landau, Fluid mechanics, Course of theoretical physics volume 6, Pergamon Press, U.K. (1987).

[21] S. Weinberg, Entropy generation and the survival of protogalaxies in an expanding universe, Astrophys. J. 168 (1971) 175 [INSPIRE].

[22] I. Muller, Zum Paradoxon der Warmeleitungstheorie, Z. Phys. 198 (1967) 329 [INSPIRE].

[23] W. Israel, Nonstationary irreversible thermodynamics: a causal relativistic theory, Annals Phys. 100 (1976) 310 [INSPIRE].

[24] W. Israel and J.M. Stewart, Transient relativistic thermodynamics and kinetic theory, Annals Phys. 118 (1979) 341 [INSPIRE].

[25] A. Muronga, Second order dissipative fluid dynamics for ultrarelativistic nuclear collisions, Phys. Rev. Lett. 88 (2002) 062302 [Erratum ibid. 89 (2002) 159901] [nucl-th/0104064] [INSPIRE]. 
[26] A. Muronga, Causal theories of dissipative relativistic fluid dynamics for nuclear collisions, Phys. Rev. C 69 (2004) 034903 [nucl-th/0309055] [INSPIRE].

[27] A. Muronga and D.H. Rischke, Evolution of hot, dissipative quark matter in relativistic nuclear collisions, nucl-th/0407114 [INSPIRE].

[28] U.W. Heinz, H. Song and A.K. Chaudhuri, Dissipative hydrodynamics for viscous relativistic fluids, Phys. Rev. C 73 (2006) 034904 [nucl-th/0510014] [INSPIRE].

[29] R. Baier, P. Romatschke and U.A. Wiedemann, Dissipative hydrodynamics and heavy ion collisions, Phys. Rev. C 73 (2006) 064903 [hep-ph/0602249] [InSPIRE].

[30] P. Romatschke and U. Romatschke, Viscosity information from relativistic nuclear collisions: how perfect is the fluid observed at RHIC?, Phys. Rev. Lett. 99 (2007) 172301 [arXiv:0706.1522] [INSPIRE].

[31] R. Baier et al., Relativistic viscous hydrodynamics, conformal invariance and holography, JHEP 04 (2008) 100 [arXiv: 0712.2451] [INSPIRE].

[32] K. Dusling and D. Teaney, Simulating elliptic flow with viscous hydrodynamics, Phys. Rev. C 77 (2008) 034905 [arXiv:0710.5932] [INSPIRE].

[33] M. Luzum and P. Romatschke, Conformal relativistic viscous hydrodynamics: applications to RHIC results at $\sqrt{s_{N N}}=200 \mathrm{GeV}$, Phys. Rev. C 78 (2008) 034915 [Erratum ibid. C 79 (2009) 039903] [arXiv:0804.4015] [INSPIRE].

[34] H. Song and U.W. Heinz, Extracting the QGP viscosity from RHIC data - A Status report from viscous hydrodynamics, J. Phys. G 36 (2009) 064033 [arXiv:0812.4274] [INSPIRE].

[35] U.W. Heinz, Early collective expansion: Relativistic hydrodynamics and the transport properties of QCD matter, Landolt-Bornstein 23 (2010) 240 [arXiv:0901.4355] [INSPIRE].

[36] B. Schenke, S. Jeon and C. Gale, Elliptic and triangular flow in event-by-event $(3+1) D$ viscous hydrodynamics, Phys. Rev. Lett. 106 (2011) 042301 [arXiv: 1009.3244] [INSPIRE].

[37] B. Schenke, S. Jeon and C. Gale, Anisotropic flow in $\sqrt{s}=2.76 \mathrm{TeV} \mathrm{Pb}+\mathrm{Pb}$ collisions at the LHC, Phys. Lett. B 702 (2011) 59 [arXiv:1102.0575] [INSPIRE].

[38] P. Bozek, Components of the elliptic flow in Pb-Pb collisions at $\sqrt{s}=2.76 \mathrm{TeV}$, Phys. Lett. B 699 (2011) 283 [arXiv:1101.1791] [INSPIRE].

[39] H. Niemi et al., Influence of the shear viscosity of the quark-gluon plasma on elliptic flow in ultrarelativistic heavy-ion collisions, Phys. Rev. Lett. 106 (2011) 212302 [arXiv:1101.2442] [INSPIRE].

[40] G.S. Denicol, J. Noronha, H. Niemi and D.H. Rischke, Origin of the Relaxation Time in Dissipative Fluid Dynamics, Phys. Rev. D 83 (2011) 074019 [arXiv:1102.4780] [InSPIRE].

[41] H. Niemi et al., Influence of a temperature-dependent shear viscosity on the azimuthal asymmetries of transverse momentum spectra in ultrarelativistic heavy-ion collisions, Phys. Rev. C 86 (2012) 014909 [arXiv:1203.2452] [INSPIRE].

[42] P. Bozek and I. Wyskiel-Piekarska, Particle spectra in Pb-Pb collisions at $\sqrt{s_{N N}}=2.76 \mathrm{TeV}$, Phys. Rev. C 85 (2012) 064915 [arXiv:1203.6513] [InSPIRE].

[43] G.S. Denicol, H. Niemi, E. Molnar and D.H. Rischke, Derivation of transient relativistic fluid dynamics from the Boltzmann equation, Phys. Rev. D 85 (2012) 114047 [Erratum ibid. D 91 (2015) 039902] [arXiv: 1202.4551] [INSPIRE]. 
[44] G.S. Denicol, E. Molnár, H. Niemi and D.H. Rischke, Derivation of fluid dynamics from kinetic theory with the 14-moment approximation, Eur. Phys. J. A 48 (2012) 170 [arXiv: 1206.1554] [INSPIRE].

[45] G.S. Denicol, S. Jeon and C. Gale, Transport coefficients of bulk viscous pressure in the 14-moment approximation, Phys. Rev. C 90 (2014) 024912 [arXiv: 1403.0962] [INSPIRE].

[46] G.S. Denicol, W. Florkowski, R. Ryblewski and M. Strickland, Shear-bulk coupling in nonconformal hydrodynamics, Phys. Rev. C 90 (2014) 044905 [arXiv: 1407.4767] [InSPIRE].

[47] A. Jaiswal, R. Ryblewski and M. Strickland, Transport coefficients for bulk viscous evolution in the relaxation time approximation, Phys. Rev. C 90 (2014) 044908 [arXiv:1407.7231] [INSPIRE].

[48] A. Jaiswal, Relativistic dissipative hydrodynamics from kinetic theory with relaxation time approximation, Phys. Rev. C 87 (2013) 051901 [arXiv:1302.6311] [INSPIRE].

[49] A. Jaiswal, Relativistic third-order dissipative fluid dynamics from kinetic theory, Phys. Rev. C 88 (2013) 021903 [arXiv: 1305.3480] [INSPIRE].

[50] W. Florkowski and R. Ryblewski, Highly-anisotropic and strongly-dissipative hydrodynamics for early stages of relativistic heavy-ion collisions, Phys. Rev. C 83 (2011) 034907 [arXiv: 1007.0130] [INSPIRE].

[51] M. Martinez and M. Strickland, Dissipative dynamics of highly anisotropic systems, Nucl. Phys. A 848 (2010) 183 [arXiv: 1007.0889] [INSPIRE].

[52] R. Ryblewski and W. Florkowski, Highly anisotropic hydrodynamics - Discussion of the model assumptions and forms of the initial conditions, Acta Phys. Polon. B 42 (2011) 115 [arXiv: 1011.6213] [INSPIRE].

[53] W. Florkowski and R. Ryblewski, Projection method for boost-invariant and cylindrically symmetric dissipative hydrodynamics, Phys. Rev. C 85 (2012) 044902 [arXiv:1111.5997] [INSPIRE].

[54] M. Martinez, R. Ryblewski and M. Strickland, Boost-invariant $(2+1)$-dimensional anisotropic hydrodynamics, Phys. Rev. C 85 (2012) 064913 [arXiv:1204.1473] [INSPIRE].

[55] R. Ryblewski and W. Florkowski, Highly-anisotropic hydrodynamics in $3+1$ space-time dimensions, Phys. Rev. C 85 (2012) 064901 [arXiv:1204.2624] [INSPIRE].

[56] D. Bazow, U.W. Heinz and M. Strickland, Second-order $(2+1)$-dimensional anisotropic hydrodynamics, Phys. Rev. C 90 (2014) 054910 [arXiv:1311.6720] [InSPIRE].

[57] L. Tinti and W. Florkowski, Projection method and new formulation of leading-order anisotropic hydrodynamics, Phys. Rev. C 89 (2014) 034907 [arXiv: 1312.6614] [INSPIRE].

[58] M. Nopoush, R. Ryblewski and M. Strickland, Bulk viscous evolution within anisotropic hydrodynamics, Phys. Rev. C 90 (2014) 014908 [arXiv:1405.1355] [INSPIRE].

[59] W. Florkowski, R. Ryblewski, M. Strickland and L. Tinti, Leading-order anisotropic hydrodynamics for systems with massive particles, Phys. Rev. C 89 (2014) 054909 [arXiv: 1403.1223] [INSPIRE].

[60] L. Tinti, Anisotropic matching principle for the hydrodynamic expansion, Phys. Rev. C 94 (2016) 044902 [arXiv: 1506. 07164] [inSPIRE].

[61] D. Bazow, U.W. Heinz and M. Martinez, Nonconformal viscous anisotropic hydrodynamics, Phys. Rev. C 91 (2015) 064903 [arXiv:1503.07443] [INSPIRE]. 
[62] D. Bazow, M. Martinez and U.W. Heinz, Transient oscillations in a macroscopic effective theory of the Boltzmann equation, Phys. Rev. D 93 (2016) 034002 [arXiv:1507.06595] [INSPIRE].

[63] M. Nopoush et al., Leading-order anisotropic hydrodynamics for central collisions, Phys. Rev. C 92 (2015) 044912 [arXiv: 1506. 05278] [INSPIRE].

[64] M. Alqahtani, M. Nopoush and M. Strickland, Quasiparticle equation of state for anisotropic hydrodynamics, Phys. Rev. C 92 (2015) 054910 [arXiv:1509.02913] [INSPIRE].

[65] E. Molnar, H. Niemi and D.H. Rischke, Derivation of anisotropic dissipative fluid dynamics from the Boltzmann equation, Phys. Rev. D 93 (2016) 114025 [arXiv: 1602.00573] [INSPIRE].

[66] E. Molnár, H. Niemi and D.H. Rischke, Closing the equations of motion of anisotropic fluid dynamics by a judicious choice of a moment of the Boltzmann equation, Phys. Rev. D 94 (2016) 125003 [arXiv: 1606.09019] [INSPIRE].

[67] M. Bluhm and T. Schäfer, Dissipative fluid dynamics for the dilute Fermi gas at unitarity: Anisotropic fluid dynamics, Phys. Rev. A 92 (2015) 043602 [arXiv:1505.00846] [INSPIRE].

[68] M. Bluhm and T. Schaefer, Model-independent determination of the shear viscosity of a trapped unitary Fermi gas: Application to high temperature data, Phys. Rev. Lett. 116 (2016) 115301 [arXiv: 1512.00862] [INSPIRE].

[69] M. Alqahtani, M. Nopoush, R. Ryblewski and M. Strickland, $(3+1) D$ quasiparticle anisotropic hydrodynamics for ultrarelativistic heavy-ion collisions, Phys. Rev. Lett. 119 (2017) 042301 [arXiv: 1703. 05808] [INSPIRE].

[70] M. Alqahtani, M. Nopoush, R. Ryblewski and M. Strickland, Anisotropic hydrodynamic modeling of 2.76 TeV Pb-Pb collisions, Phys. Rev. C 96 (2017) 044910 [arXiv:1705.10191] [INSPIRE].

[71] D. Almaalol and M. Strickland, Anisotropic hydrodynamics with a scalar collisional kernel, Phys. Rev. C 97 (2018) 044911 [arXiv:1801.10173] [InSPIRE].

[72] D. Almaalol, M. Alqahtani and M. Strickland, Anisotropic hydrodynamic modeling of 200 GeV Au-Au collisions, Phys. Rev. C 99 (2019) 044902 [arXiv: 1807.04337] [InSPIRE].

[73] W. Florkowski, R. Maj, R. Ryblewski and M. Strickland, Hydrodynamics of anisotropic quark and gluon fluids, Phys. Rev. C 87 (2013) 034914 [arXiv:1209.3671] [INSPIRE].

[74] W. Florkowski, E. Maksymiuk, R. Ryblewski and L. Tinti, Anisotropic hydrodynamics for a mixture of quark and gluon fluids, Phys. Rev. C 92 (2015) 054912 [arXiv:1508.04534] [INSPIRE].

[75] W. Florkowski, E. Maksymiuk and R. Ryblewski, Anisotropic-hydrodynamics approach to a quark-gluon fluid mixture, Phys. Rev. C 97 (2018) 014904 [arXiv:1711.03872] [INSPIRE].

[76] D. Almaalol, M. Alqahtani and M. Strickland, Anisotropic hydrodynamics with number-conserving kernels, Phys. Rev. C 99 (2019) 014903 [arXiv:1808.07038] [inSPIRE].

[77] J.D. Bjorken, Highly relativistic nucleus-nucleus collisions: the central rapidity region, Phys. Rev. D 27 (1983) 140 [INSPIRE].

[78] A. Bialas and W. Czyz, Boost Invariant Boltzmann-Vlasov equations for relativistic quark-anti-quark plasma, Phys. Rev. D 30 (1984) 2371 [INSPIRE].

[79] A. Białas and W. Czyż, Oscillations of quark-gluon plasma generated in strong color fields, Nucl. Phys. B 296 (1988) 611. 
[80] W. Florkowski, R. Ryblewski and M. Strickland, Anisotropic hydrodynamics for rapidly expanding systems, Nucl. Phys. A 916 (2013) 249 [arXiv:1304.0665] [InSPIRE].

[81] W. Florkowski, R. Ryblewski and M. Strickland, Testing viscous and anisotropic hydrodynamics in an exactly solvable case, Phys. Rev. C 88 (2013) 024903 [arXiv: 1305.7234] [INSPIRE].

[82] P. Romatschke and M. Strickland, Collective modes of an anisotropic quark gluon plasma, Phys. Rev. D 68 (2003) 036004 [hep-ph/0304092] [InSPIRE].

[83] M. Strickland, http://personal.kent.edu/ mstrick6/code/ (2017).

[84] M. Martinez and M. Strickland, Measuring QGP thermalization time with dileptons, Phys. Rev. Lett. 100 (2008) 102301 [arXiv:0709.3576] [INSPIRE].

[85] M. Martinez and M. Strickland, Pre-equilibrium dilepton production from an anisotropic quark-gluon plasma, Phys. Rev. C 78 (2008) 034917 [arXiv:0805.4552] [INSPIRE].

[86] M. Martinez and M. Strickland, Constraining relativistic viscous hydrodynamical evolution, Phys. Rev. C 79 (2009) 044903 [arXiv: 0902.3834] [INSPIRE].

[87] G.S. Denicol, T. Koide and D.H. Rischke, Dissipative relativistic fluid dynamics: a new way to derive the equations of motion from kinetic theory, Phys. Rev. Lett. 105 (2010) 162501 [arXiv: 1004.5013] [INSPIRE].

[88] A. Kurkela and A. Mazeliauskas, Chemical equilibration in weakly coupled QCD, Phys. Rev. D 99 (2019) 054018 [arXiv: 1811.03068] [INSPIRE].

[89] A. Kurkela and A. Mazeliauskas, Chemical equilibration in hadronic collisions, Phys. Rev. Lett. 122 (2019) 142301 [arXiv: 1811.03040] [INSPIRE]. 